\title{
Systemic Therapy of Advanced Renal Cell Carcinoma- Summary of Main Presentations at the ASCO, ASCO-GU and ESMO 2012 Annual Meetings
}

\author{
Andres Jan Schrader \\ Department of Urology, Ulm Medical Center, Ulm, Germany \\ Email: ajschrader@gmx.de
}

Received November 12, 2012; revised December 28, 2012; accepted January 17, 2013

\begin{abstract}
A number of very interesting studies presented this year at the ASCO (American Society of Clinical Oncology) Annual Meeting, the ASCO-GU (ASCO Genitourinary Cancers) Spring Meeting, and the ESMO (European Society for Medical Oncology) Annual Congress could strongly influence or even revolutionize the systemic treatment of advanced renal cell carcinoma (RCC). The aim of this article is to identify, summarize and discuss some outstanding studies of direct or indirect clinical relevance for systemic therapy.
\end{abstract}

Keywords: Renal Cell Carcinoma; ASCO; ESMO; Review; Systemic Therapy

\section{Introduction}

Renal cell carcinoma (RCC) is the third most common urologic malignancy after prostatic and urothelial cancer. The treatment of localized RCC has not changed significantly in the last 12 months. Its three main aims remain the same: maximum tumor control, low perioperative morbidity, and maximum preservation of renal function. Thus partial nephrectomy (i.e. nephron sparing surgery), when oncologically justified and technically feasible, is the standard treatment for organ-confined kidney tumors [1]. Local ablation procedures (cryotherapy, radiofrequency ablation) are conceivable alternatives for small $(<3$ $\mathrm{cm}$ ) peripheral tumors in patients who have a markedly increased perioperative risk or who appear to have a limited life expectancy and those who refuse an "active surveillance" strategy. At present, the decision to offer neoadjuvant systemic therapy should be made on a case by case basis in patients with technically nonresectable tumors, only [2].

Even though metastatic RCC patients still have no realistic chance of a cure, several treatment options have yet become available: a series of multikinase inhibitors (sunitinib, pazopanib, sorafenib, axitinib, and probably also tivozanib very shortly), mTOR inhibitors (temsirolimus, everolimus), a monoclonal antibody (bevacizumab), and of course the still sparadically used nonspecific immunotherapeutic agents interferon-alpha (IFN-alpha) and interleukin 2 (IL-2). These agents, precisely when applied in sequence, have demonstrated their potential to significantly delay the course of the disease [3]. More- over, this wide selection of agents can, at least theoreticcally, help to individualize treatment, alleviate tumorrelated symptoms, and thus improve the prognosis of metastatic RCC [4].

Once again, the many fascinating new studies on the treatment of advanced RCC presented at the 2012 Annual Meetings of the American Society of Clinical Oncology (ASCO), the ASCO-GU (ASCO Genitourinary Cancers) Spring Meeting, and the Annual Meeting of the European Society for Medical Oncology (ESMO) clearly reflect the continued high level of interest in this disease as well as in its role as a model tumor. Since significant new insights have been gained here rather than in the treatment of localized RCC, the aim of this article is to identify, summarize and discuss some outstanding studies of direct or indirect clinical relevance for systemic RCC therapy.

\section{Cardiotoxicity of Targeted Systemic Therapy}

Targeted therapy is currently the standard of care in metastatic RCC. By the same token, a number of studies published during recent years have been concerned with evaluating the potential cardiotoxicity of these targeted agents, especially that of multikinase inhibitors [5,6]. At the ASCO Annual Meeting this year, Hall et al. [7] from Stanford presented a study that retrospectively analyzed the use of targeted drugs for treatment of 159 RCC patients between 2004 and 2010. Cardiovascular event rates observed in patients treated with these agents were 
surprisingly high and ranged between $51 \%$ and $68 \%$ for the tyrosine kinase inhibitors as well as for bevacizumab. A relatively small number of patients received mTOR inhibitors, but cardiovascular events/toxicity rates appeared to be lower here $(33 \%-38 \%)$. The percentage of patients with decreased left ventricular ejection fraction (LVEF) was also surprisingly high (14\% on the average). The authors concluded that cardiotoxicity and cardiovascular events are frequently observed during targeted therapy and often have clinical relevance and that close cardiac monitoring is necessary for early detection.

Another large prospective study on this topic was presented by Haas et al. [8]. Here too, the aim was to determine what types and degrees of cardiotoxicity may be expected with tyrosine kinase inhibitors (TKI). They prospectively evaluated the 1292 patients treated with sunitinib, sorafenib or placebo on an outpatient basis during the ECOG 2805 (ASSURE) trial. In contrast to the study by Hall et al. [7], however, this study included only patients without primary heart disease; they were examined by MUGA (multigated acquisition) scans every three months. In this study, the rate of patients with an LVEF decrease of at least $16 \%$ was only $2.3 \%$ with sunitinib, $1.8 \%$ with sorafenib, and $1.0 \%$ in the placebo arm. Virtually all decreases in LVEF were reversible. Only five patients had myocardial ischemic events in the course of the study. The authors concluded that the risk of significant cardiovascular toxicity during systemic TKI therapy is at most only slightly elevated, at least in patients without primary heart disease.

\section{New Multikinase Inhibitors: Effectiveness, Dose Finding, and Predictive Clinical Factors}

Motzer et al. [9] presented final results from the phase III TIVO-1 trial, in which tivozanib (AV-951), a highly potent new vascular endothelial growth factor receptor (VEGFR) targeting TKI, was prospectively tested against sorafenib. Tivozanib was applied at the standard dose of $1.5 \mathrm{mg}$ a day. All patients in this study suffered from clear cell RCC, had undergone nephrectomy, and had not received any prior $\operatorname{VEGF(R)-~or~mTOR-targeted~therapy.~}$ The objective response rate was $33 \%$ in the tivozanib arm $(n=260)$ and $23 \%$ in the sorafenib arm; another $52 \%$ and $65 \%$ achieved disease stabilization. Median progression-free survival was 11.9 months for tivozanib and 9.1 months for sorafenib (HR $0.8, p=0.04$ ). Toxicity in the two treatment arms was comparable in many respects. However, patients treated with tivozanib had a higher incidence of arterial hypertension (44\% vs. 34\%) and dysphonia $(21 \%$ vs. $5 \%)$, while those who received sorafenib suffered more often from diarrhea $(22 \%$ vs. $32 \%$ ), showed palmar or plantar erythema (13\% vs. $54 \%$ ), and reported alopecia ( $2 \%$ vs. $21 \%$ ). The good tolerance of tivozanib is underscored by the fact that it necessitated significantly fewer dose reductions and toxicity-related treatment interruptions than sorafenib (12\% vs. $43 \%$ and $18 \%$ vs. $35 \%$ ).

The most common side effect or accompanying symptom in patients receiving tivozanib was hypertension. Here an analysis was done to determine whether the blood pressure increase during therapy correlated with the treatment response. It was clearly demonstrated that patients who reacted with an increase in blood pressure had a significantly longer median progression-free survival than those who showed no increase in their diastolic or systolic blood pressure during therapy. Thus the median progression-free survival was 18.3 months in patients with a diastolic blood pressure of $>90 \mathrm{mmHg}$ during tivozanib treatment but only 9.1 months in the control group $(\leq 90 \mathrm{mmHg})(\mathrm{HR} 0.55 ; 95 \% \mathrm{KI} 0.38-0.78$; $\mathrm{p}$ $=0.001)$. Motzer et al. [9] concluded that a blood pressure increase in patients receiving tivozanib could be an indicator or predictive factor for the individual effectiveness of the agent. Tivozanib is expected to gain approval for treatment of metastatic RCC.

Rini et al. [10] analyzed the efficacy and pharmacokinetics of the multikinase inhibitor axitinib as a first-line agent after having demonstrated its effectiveness as second-line therapy in a phase III trial last year [11]. The basis for the current phase II study presented in 2012 was previous evidence that, when applying a constant dose of this oral TKI, the plasma level can vary individually and can again significantly increase after dose titration/escalation. Moreover, patients with a low plasma level had shorter progression-free survival times.

The 213 patients treated in the phase II study presented here received axitinib at a starting dose of $5 \mathrm{mg}$ twice a day. The dose could potentially be increased in patients who did not have a significant blood pressure elevation $(>150 / 90 \mathrm{mmHg})$ or grade $3 / 4$ side effects. The first result of the study was that patients primarily excluded from dose escalation due to treatment-emergent toxicity already showed a primary tendency towards longer progression-free survival times than those with fewer side effects during axitinib treatment (16.4 vs. 14.5 months). The response rate also appeared to be higher among patients who had side effects than among those with no complications. Accordingly, patients with side effects had significantly higher axitinib plasma levels than those without significant toxicity (AUC12: $234 \mathrm{vs.} 99 \mathrm{ng} \cdot \mathrm{h} / \mathrm{mL}$; $\mathrm{p}<0.0001)$. Rini et al. [10] then once again differentiated the response in the groups, which showed an AUC12 $\geq 150 \mathrm{ng} \cdot \mathrm{h} / \mathrm{mL}$ vs. $<150 \mathrm{ng} \cdot \mathrm{h} / \mathrm{mL}$ after 14 days of treatment. Here there were also clear advantages for the group with the higher plasma level: progression-free survival of 13.9 vs. 11.0 months and an objective response rate of $59 \%$ vs. $40 \%$. As already described for tivozanib 
in the TIVO-1 study [9], hypertension proved to be a significant drug response parameter here too. Thus the conclusion was also drawn for axitinib that hypertension could be a good indicator of TKI response. Moreover, the blood pressure increase correlated with the serum concentration of the drug. The authors concluded that patients must reach a certain plasma level of TKI (here explicitly axitinib) that can otherwise vary interindividually during continuous standard-dose treatment. The TKI dosage could/should thus be individualized in the future to achieve optimal treatment results.

Similar preliminary results indicating that a dose escalation can increase the effectiveness of TKI were already reported for sorafenib several years ago [12-14]. Thus the results presented by Rini et al. [10] on enhancing the dose and thus the effectiveness of axitinib shed a somewhat different light on the results of the AXIS trial, in which dose escalation was only permitted in the axitinib arm [11].

\section{Blockade of the HGF/MET Signal Transduction Pathway-Future Prospects?}

Various studies from 2012 dealt with the question of whether inhibition of HGF/MET signal transduction (a possible escape mechanism during VEGF-targeted therapy) may be an effective modality for managing pretreated metastatic RCC. Puzanov et al. [15] presented a small phase I study $(\mathrm{n}=20)$ that evaluated response to sorafenib combined with tivantinib (ARQ 197, a MET blocker). Seventy percent of the patients had been pretreated, and eighty percent had clear cell RCC. This combined therapy achieved a median freedom from progression of 12.7 months, at least in patients with conventional histology. However, a correlation was not found in all cases between the response to this combination and the MET expression detected in the primary tumor.

Patients with pretreated metastatic RCC received cabozantinib $(140 \mathrm{mg} / \mathrm{d})$ in a second study on this topic presented by Choueiri et al. [16]. Cabozantinib (XL 184) is another TKI, but it blocks both, the VEGF and HGF/ MET signal transduction pathways. Again, the underlying aim was to overcome resistance to VEGF-targeted therapy and to effectively treat the RCC sub-group that appears to be primarily refractory to such therapy (approx. $26 \%$ of all patients [4]). The therapeutic effectiveness of blocking both the HGF/MET and VEGF signal transduction pathways has already been demonstrated for other tumors: prostate cancer [17], thyroid cancer [18,19], hepatocellular carcinoma [20], breast cancer [21], and ovarian cancer [22]. In the study by Choueiri et al. [16], cabozantinib was given to 25 in part heavily pretreated patients with progressive RCC. VEGF-targeted therapy had already been performed in $88 \%$, mTOR-targeted therapy in $60 \%$, and both in $52 \%$ of all patients. This small special population treated with cabozantinib had a response rate of $28 \%$, and disease stabilization was observed in another $52 \%$ of all patients. Only one patient $(4 \%)$ had a primarily progressive tumor. The median progression-free survival time was 14.7 months. Toxicity in this patient population was roughly comparable to that of TKI already approved for RCC and other cancers.

Results of a phase II study on foretinib, another MET/ VEGFR-2 inhibitor, in papillary RCC were just as promising [23].

\section{Patient Preference-Effectiveness in First-Line Therapy}

One of the first studies to assess the preference of urooncologic patients as the primary endpoint was presented by Bernard Escudier et al. [24] at the ASCO Annual Meeting 2012. The PISCES study compared the sequence of pazopanib/sunitinib, applied for 10 weeks each, with the reverse sequence (sunitinib/pazopanib). The aim was to evaluate whether possible differences between the agents with regard to their tolerance and side effect profile were relevant for the patients and to determine which drug the patients preferred at the end of the sequence. There were 82 patients in the sunitinib/pazopanib arm and 86 in the pazopanib/sunitinibarm. In the end, pazopanib was preferred by $70 \%$ and sunitinib by $22 \%$ of the patients whose assessment could ultimately be included in the analysis. Eight percent expressed no preference. The attending physicians were also questioned, and the results were comparable ( $61 \%$ vs. $22 \%$ vs. $17 \%)$. The main reasons patients gave for preferring pazopanib were a better quality of life and less frequent or milder occurrence of fatigue symptoms, taste changes, oral mucosal lesions, nausea and vomiting, hand-foot syndrome, loss of appetite, and diarrhea. The latter was surprising, since concomitant data indicated that diarrhea was at least numerically more frequent with pazopanib than with sunitinib (42\% vs. 32\%). Similarly, nausea (33\% vs. $30 \%)$ and loss of appetite (20\% vs. $19 \%)$ were not recorded less often with pazopanib than with sunitinib. Thus an important study outcome was recognition of the need to further evaluate why the patient-perceived quality-of-life impairment caused by certain side effects did not correlate in all cases with the frequency and degree of the physician-recorded complications during treatment with the various agents. Dose reductions were necessary in $13 \%$ of patients treated with pazopanib and in $20 \%$ treated with sunitinib.

The COMPARZ study $(\mathrm{n}=1110)$ also directly compared the two agents pazopanib and sunitinib for their effectiveness and toxicity in first-line treatment. Motzer et al. [25] presented the first results of this phase III trial at the ESMO Annual Congress in Vienna. Treatment 
with pazopanib $(800 \mathrm{mg} / \mathrm{d})$ and sunitinib $(50 \mathrm{mg} / \mathrm{d}, \mathrm{d} 1-28$, q42) resulted in comparable progression-free survival times (external validation: 8.4 vs. 9.5 months, HR 1.05; local validation: 10.5 vs. 10.2 months, HR 1.0 ) and no significant differences in overall survival (28.4 vs. 29.3 months, HR $0.91, \mathrm{p}=0.28$ ). Dose reductions and toxicity-related treatment interruptions were necessary in $44 \%$ and $24 \%$ of all cases with pazopanib and in $51 \%$ and $19 \%$ with sunitinib; thus the two agents were comparable in this respect as well. Elevated liver enzymes and hair color changes were seen more often in patients treated with pazopanib, while bone marrow suppression, handfoot syndrome, and fatigue were more common in those treated with sunitinib. Both groups had virtually the same incidence of grade 3/4 toxicity. However, quality-of-life data collected on day 28 of each cycle showed clear advantages for pazopanib.

\section{Immunotherapy-Future Prospects?}

After two decades of using nonspecific immunotherapeutic agents (largely IFN- $\alpha$ and IL-2) to treat metastatic RCC with only moderate success [26], immunotherapy was de facto abandoned as soon as targeted agents were introduced.

BMS-936558 is a fully human monoclonal antibody directed against PD-1 (programmed death 1). The PD-1/ PD ligand 1 (PD-L1) system contributes to deactivation of $T$ cells [27]. High expression of PD-1/PD-L1 was found to correlate with a poorer prognosis and a more aggressive tumor biology in patients with RCC [28]. At the last ASCO Annual Meeting, McDermott et al. [29] presented a current study on the effectiveness and safety of the anti-PD-1 antibody BMS-936558 that also included 34 patients with pretreated RCC [29,30]. Patients were treated four times at 2-week intervals; responders could continue treatment. Antiangiogenetic therapy had already been given to $74 \%$ of the RCC patients, and $59 \%$ had even received nonspecific immunotherapy. Side effects of BMS-936558 were generally mild. Fatigue occurred most frequently (38\%); typical for an immunotherapeutic agent was the skin reaction seen $24 \%$ of all cases. Application of the antibody elicited partial remission in $27 \%$ of the patients. The success of treatment did not appear to be critically dependent on the dosage (1 or $10 \mathrm{mg} / \mathrm{kg} \mathrm{BW}$ ). Particularly striking was the fact that some lesions responded significantly to treatment with BMS-936558, while new metastases developed at the same time. This too is known from the era of cytokinebased immunotherapy. It was also evident that, although treatment was stopped after 96 weeks in all patients, some of them still remained stable for long periods of time, another great advantage of immunotherapy over treatment with VEGF- or mTOR-targeted agents. In addition, a subpopulation of the patients could be evaluated to determine whether their primary tumor was PD-L1positive. Here a treatment response was not observed in any of the patients with PD-L1-negative tumors, but it was seen in over a third of those whose primary tumors expressed PD-L1. This too might be helpful in making a decision regarding treatment with the antibody BMS936558. In the next few years, we will be eagerly awaiting further results from trials assessing its clinical benefit as monotherapy or in combination with other targeted agents.

\section{Combinations and Sequences}

The INTORACT study (phase IIIb) presented by Rini et al. [31] at the ESMO Annual Congress in Vienna compared the combinations of temsirolimus (TEMSR)/bevacizumab and IFN/bevacizumab for their effectiveness and toxicity in 791 non-pretreated patients with clear cell RCC. Ninety-two percent of all patients had a good to intermediate MSKCC risk score, and eighty-five percent had undergone primary nephrectomy. In short, the study showed that the two groups were comparable with regard to progression-free $(9.1$ vs. 9.3 months, $\mathrm{p}=0.76)$ and overall survival ( 25.8 vs. 25.5 months, $\mathrm{p}=0.64)$, but TEMSR/bevacizumab tended to show higher toxicity.

A great deal of attention was also paid to the presentation of results from the phase III INTORSECT ("404") trial, in which 512 patients received TEMSR or sorafenib after sunitinib failure [32]. Eighty-six percent of the patients had undergone primary nephrectomy and eightythree percent suffered from clear cell RCC; over $2 / 3$ of all patients had an intermediate risk according to MSKCC criteria. Patients treated with TEMSR showed an insignificant progression-free survival (PFS) advantage ( 4.3 vs. 3.9 months; $p=0.19$ ), whereas those who received sorafenib as second-line therapy appeared to live significantly longer $(12.3$ vs. 16.6 months; $p=0.014)$. The previous response to sunitinib did not predict whether patients were more likely to profit from an mTOR inhibitor or another TKI as second-line therapy. The two agents differed with regard to the type, but not the intensity or frequency of toxicity in a second-line setting.

\section{Does Progression-Free Survival Predict Overall Survival with Targeted Therapy?}

The above-mentioned results of the INTORSECT study once again raised the question of whether progressionfree survival (PFS) can or should be regarded as a good study endpoint or predictor of overall survival. In the INTORSECT study, TEMSR had a slight PFS advantage (4.3 vs. 3.9 months; $p=0.19$ ), though sorafenib resulted in a significantly longer overall survival (12.3 vs. 16.6 months; $\mathrm{p}=0.014$ ).

The AXIS study yielded similarly surprising survival 
data that were also presented at the ESMO Annual Congress [33]. Here axitinib was clearly superior to its competitor sorafenib in terms of PFS (6.7 vs. 4.7 months; $p<$ 0.001 ) [11], but the two agents did not differ with regard to overall survival (20.1 vs. 19.2 months; HR 0.97, $\mathrm{p}=$ 0.37) [33]. Even the subgroup of cytokine-pretreated patients, where the PFS differed most markedly (12.1 vs. 6.5 months; $\mathrm{p}<0.001)$, showed no difference in overall survival (29.4 vs. 27.8 months; HR $0.81,95 \%$ CI 0.56 1.19).

Thus "survival" should perhaps again become the preferred primary endpoint in the future, and more emphasis should be placed on the quality of life in the time remaining (i.e., until death) with continued palliative care.

\section{Patient Selection and Treatment Duration}

In many cases, the results obtained in prospective phase III trials cannot be reproduced in the reality of everyday practice. This is probably due not least to a specific selection of patients that qualify for participation in these so-called "pivotal clinical trials". Heng et al. [34] retrospectively evaluated patient populations treated with VEGF-targeted agents (sunitinib, sorafenib, pazopanib, axitinib, and bevacizumab) at 17 American or British centers. Of the 2076 metastatic RCC patients identified, 1182 could have been treated in most phase III trials (applying the so-called "standard inclusion criteria"). In other words, they would have essentially qualified for participation in a phase III trial on the basis of their general condition, laboratory parameters, histological subtype, nephrectomy status, and absence of CNS metastases. 894 patients were retrospectively classified as "ineligible for the study." The comparison of the two patient groups presented by Heng et al. [34] showed not only that theoretically eligible patients had a significantly higher response rate $(30 \%$ vs. $21 \%)$ and lived significantly longer ( 28.8 vs. 14.5 months), but also that, even using multivariate analysis, "eligibility" correlated with a significantly better prognosis. This analysis impressively demonstrated that results from phase III trials cannot be fully applied to the total patient population and that valuable insights should and must also (additionally) be gained from registries and phase IV analyses.

Ferte et al. [35] convincingly showed that the tumor growth rate increases exponentially after discontinuing systemic VEGF- or mTOR-targeted therapy with sorafenib $(n=84)$ or everolimus $(n=52)$. The authors argue that quick discontinuation of targeted therapy should be reconsidered also in cases of slow but clinically insignificant progression.

\section{Summary}

To date, none of the new targeted agents have shown curative potential as either monotherapy or combination therapy. However, the increasingly broad range of new agents enables us to achieve prolonged stable disease in many patients with metastatic RCC and to tailor treatment sequences to the individual patients and their tumors, though predictive parameters are still lacking. Axitinib was just approved, and tivozanib is expected to gain approval. Immunotherapy research appears to have once again brought forth a promising new agent, the anti-PD-1 antibody BMS-936558. Clinical symptoms during TKI therapy, particularly hypertension, could indicate high effectiveness or a sufficient plasma activity level. Accordingly, the latter may soon play a decisive role in treatment monitoring.

\section{REFERENCES}

[1] A. J. Schrader and S. Steffens, "Renal Cell Carcinoma Update: News from the AUA, EAU, and ASCO Annual Meetings 2011," ISRN Urology, Vol. 2012, 2012, Article ID: 748235. doi: $10.5402 / 2012 / 748235$

[2] A. J. Schrader, S. Steffens, T. J. Schnoeller, M. Schrader and M. A. Kuczyk, "Neoadjuvant Therapy of Renal Cell Carcinoma: A Novel Treatment Option in the Era of Targeted Therapy?" International Journal of Urology, Vol. 19, No. 10, 2012, pp. 903-907. doi:10.1111/j.1442-2042.2012.03065.x

[3] U. Harmenberg, S. Lundstam, T. Wahlgren, J. Kowalski, M. Jakobsson, R. Sandin, B. Ljungberg and P. Sandström, "Treatment and Overall Survival (OS) in Metastatic Renal Cell Carcinoma (mRCC): A Swedisch PopulationBased Study (2000-2008)," Journal of Clinical Oncology, Vol. 30, Suppl. 5, 2012, Abstract No. 389.

[4] D. Y. Heng, W. Xie, M. M. Regan, T. Cheng, S. North, J. J. Knox, C. Kollmannsberger, D. McDermott, B. I. Rini and T. K. Choueiri, "Prognostic Factors for Overall Survival (OS) in Patients with Metastatic Renal Cell Carcinoma (RCC) Treated with Vascular Endothelial Growth Factor (VEGF)-Targeted Agents: Results from a Large Multicenter Study," Journal of Clinical Oncology, Vol. 27, No. 34, 2009, pp. 5794-5799.

[5] M. Schmidinger, C. C. Zielinski, U. M. Vogl, A. Bojic, M. Bojic, C. Schukro, M. Ruhsam, M. Hejna and H. Schmidinger, "Cardiac Toxicity of Sunitinib and Sorafenib in $\mathrm{Pa}-$ tients with Metastatic Renal Cell Carcinoma," Journal of Clinical Oncology, Vol. 26, No. 32, 2008, pp. 5204-5212. doi:10.1200/JCO.2007.15.6331

[6] M. Schmidinger, U. M. Vogl, C. Schukro, A. Bojic, M. Bojic, H. Schmidinger and C. C. Zielinski, "Cardiac Involvement in Patients with Sorafenib or Sunitinib Treatment for Metastatic Renal Cell Carcinoma," Journal of Clinical Oncology, Vol. 25, No. 18S, 2007, Abstract No. 5110.

[7] P. S. Hall, R. Witteles, S. Srinivas and L. C. Harshman, "Incidence and Severity of Cardiotoxicity in Metastatic Renal Cell Carcinoma (RCC) Patients Treated with Targeted Therapies," Journal of Clinical Oncology, Vol. 30, 2012, Abstract No. 4610.

[8] N. B. Haas, J. Manola, B. Ky, K. T. Flaherty, R. Uzzo, C. 
G. Wood, C. J. Kane, M. A. S. Jewett, M. B. Atkins, J. P. Dutcher and R. S. DiPaola, "Cardiac Safety Analysis for a Phase III Trial of Sunitinib (SU) or Sorafenib (SO) or Placebo (PLC) in Patients (pts) with Resected Renal Cell Carcinoma (RCC)," Journal of Clinical Oncology, Vol. 30, 2012, Abstract No. 4500.

[9] R. J. Motzer, D. Nosov, T. Eisen, I. N. Bondarenko, V. Lesovoy, O. N. Lipatov, P. Tomczak, A. A. Lyulko, A. Alyasova, M. Harza, M. Kogan, B. Y. Alexeev, C. N. Sternberg, C. Szczylik, J. Zhang, A. L. Strahs, B. Esteves, W. J. Slichenmyer, A. Berkenblit and T. E. Hutson, "Tivozanib versus Sorafenib as Initial Targeted Therapy for Patients with Advanced Renal Cell Carcinoma: Results from a Phase III Randomized, Open-Label, Multicenter Trial," Journal of Clinical Oncology, Vol. 30, 2012, Abstract No. 4501.

[10] B. I. Rini, V. Gruenwald, M. N. Fishman, B. Melichar, T. Ueda, P. A. Karlov, A. H. Bair, Y. Chen, S. Kim and E. Jonasch, "Axitinib for First-Line Metastatic Renal Cell Carcinoma (mRCC): Overall Efficacy and Pharmacokinetic (PK) Analyses from a Randomized Phase II Study.," Journal of Clinical Oncology, Vol. 30, 2012, Abstract No. 4503.

[11] B. I. Rini, B. Escudier, P. Tomczak, A. Kaprin, T. E. Hutson, C. Szczylik, J. C. Tarazi, B. Rosbrook, S. Kim and R. J. Motzer, "Axitinib versus Sorafenib as SecondLine Therapy For Metastatic Renal Cell Carcinoma (mRCC): Results of Phase III AXIS Trial.," Journal of Clinical Oncology, Vol. 29, Suppl. 15, 2011, Abstract No. 4503.

[12] M. Staehler, N. Haseke, T. Stadler, K. Zilinberg, C. Nordhaus, M. Dieckmann, P. Nuhn and C. G. Stief, "Intraindividual Sorafenib Dose Escalation in the Treatment of Metastatic Clear Cell Renal Cell Carcinoma (mRCC)," Annual Meeting of the German Society for Urology (DGU), Vol. 61, 2009, Abstract No. P 6.10.

[13] R. J. Amato, J. Jac, P. Harris, M. Dalton, S. Saxena, F. Monzon, J. Zhai, J. Brady and J. P. Willis, "A Phase II Trial of Intra-Patient Dose Escalated-Sorafenib in Patients (pts) with Metastatic Renal Cell Cancer (MRCC)," Journal of Clinical Oncology, Vol. 26, No. 15S, 2008, Abstract No. 5122.

[14] C. Szczylik, T. Demkow, M. Staehler, F. Rolland, S. Negrier, T. E. Hutson, R. M. Bukowski, U. J. Scheuring, K. Burk and B. Escudier, "Randomized Phase II Trial of First-Line Treatment with Sorafenib Versus Interferon in Patients with Advanced Renal Cell Carcinoma: Final Results," Journal of Clinical Oncology, Vol. 25, No. 18S, 2007, Abstract No. 5025.

[15] I. Puzanov, J. A. Sosman, A. Santoro, R. E. Martell, G. K. Dy, L. Williams Goff, W. W. Ma, G. J. Fetterly, S. A. Michael, J. A. Means-Powell, F. Chai, M. Lamar, G. M. Strauss, P. A. Zucali, W. M. Chiang, J. Jarboe, B. E. Schwartz and A. A. Adjei, "Safety and Efficacy of MET Inhibitor Tivantinib (ARQ 197) Combined with Sorafenib in Patients (pts) with Renal Cell Carcinoma (RCC) from a Phase I Study," Journal of Clinical Oncology, Vol. 30, 2012, Abstract No. 4545.

[16] T. K. Choueiri, S. K. Pal, D. F. McDermott, D. A. Ramies, S. Morrissey, Y. Lee, D. Miles, J. Holland and J. P.
Dutcher, "Efficacy of Cabozantinib (XL184) in Patients (pts) with Metastatic, Refractory Renal Cell Carcinoma (RCC)," Journal of Clinical Oncology, Vol. 30, 2012, Abstract No. 4504.

[17] M. R. Smith, C. Sweeney, D. E. Rathkopf, H. I. Scher, C. Logothetis, D. J. George, C. S. Higano, E. Y. Yu, A. L. Harzstark, E. J. Small, A. O. Sartor, M. S. Gordon, N. J. Vogelzang, D. C. Smith, M. Hussain, J. S. De Bono, N. B. Haas, C. Scheffold, Y. Lee and P. G. Corn, "Cabozantinib (XL184) in Chemotherapy-Pretreated Metastatic Castration Resistant Prostate Cancer (mCRPC): Results from a Phase II Nonrandomized Expansion Cohort (NRE)," Journal of Clinical Oncology, Vol. 30, 2012, Abstract No. 4513.

[18] M. E. Cabanillas, M. S. Brose, D. A. Ramies, Y. Lee, D. Miles and S. I. Sherman, "Antitumor Activity of Cabozantinib (XL184) in a Cohort of Patients (pts) with Differentiated Thyroid Cancer (DTC)," Journal of Clinical Oncology, Vol. 30, 2012, Abstract No. 5547.

[19] P. Schoffski, R. Elisei, S. Müller, M. S. Brose, M. H. Shah, L. F. Licitra, B. Jarzab, V. Medvedev, M. Kreissl, B. Niederle, E. E. W. Cohen, L. J. Wirth, H. Y. Ali, C. Hessel, Y. Yaron, D. W. Ball, B. Nelkin, S. I. Sherman and M. Schlumberger, "An International, Double-Blind, Randomized, Placebo-Controlled Phase III Trial (EXAM) of Cabozantinib (XL184) in Medullary Thyroid Carcinoma (MTC) Patients (pts) with Documented RECIST Progression at Baseline," Journal of Clinical Oncology, Vol. 30, 2012, Abstract No. 5508.

[20] C. Verslype, A. L. Cohn, R. K. Kelley, T. S. Yang, W. C. Su, D. A. Ramies, Y. Lee, X. Shen and E. Van Cutsem, "Activity of Cabozantinib (XL184) in Hepatocellular Carcinoma: Results from a Phase II Randomized Discontinuation Trial (RDT)," Journal of Clinical Oncology, Vol. 30, 2012, Abstract No. 4007.

[21] E. P. Winer, S. Tolaney, H. Nechushtan, R. Berger, R. Kurzrock, I. G. Ron, P. Schoffski, A. Awada, C. A. Yasenchak, H. A. Burris, D. A. Ramies, T. Rafferty and X. Shen, "Activity of Cabozantinib (XL184) in Metastatic Breast Cancer (MBC): Results from a Phase II Randomized Discontinuation Trial (RDT)," Journal of Clinical Oncology, Vol. 30, 2012, Abstract No. 535.

[22] R. J. Buckanovich, R. Berger, A. Sella, B. I. Sikic, X. Shen, D. A. Ramies, D. C. Smith and I. B. Vergote, "Activity of Cabozantinib (XL184) in Advanced Ovarian Cancer Patients (pts): Results from a Phase II Randomized Discontinuation Trial (RDT)," Journal of Clinical Oncology, Vol. 29, 2011, Abstract No. 5008.

[23] T. K. Choueiri, U. N. Vaishampayan, J. E. Rosenberg, T. Logan, A. L. Harzstark, B. I. Rini, S. Srinivas, L. M. Adams, L. J. Sherham, L. H. Ottesen, D. F. McDermott, D. Bottaro, W. M. Linehan and R. Srinivasan, "A Phase II and Biomarker Study (MET 111644) of the Dual Met/ VEGFR-2 Inhibitor Foretinib in Patients with Sporadic and Hereditary Papillary Renal Cell Carcinoma: Final Efficacy, Safety, and PD Results," Journal of Clinical Oncology, Vol. 30, No. 5S, 2012, Abstract No. 355.

[24] B. J. Escudier, C. Porta, P. Bono, U. de Giorgi, O. Parikh, R. E. Hawkins, E. Sevin, S. Negrier, S. Khan, L. McCann, F. Mehmud and C. Cella, "Patient Preference between 
Pazopanib (Paz) and Sunitinib (Sun): Results of a Randomized Double-Blind, Placebo-Controlled, Cross-Over Study in Patients with Metastatic Renal Cell Carcinoma (mRCC)-PISCES Study, NCT 01064310," Journal of Clinical Oncology, Vol. 30, 2012, Abstract No. CRA4502.

[25] R. Motzer, T. E. Hutson, J. Reeves, R. Hawkins, J. Guo, P. Nathan, M. Staehler, P. de Souza, J. R. Merchan, K. Fife, J. Jin, R. Jones, H. Uemura, U. de Giorgi, U. Harmenberg, J. Wang, D. Cella, L. McCann, K. Deen and T. K. Choueiri, "Randomized, Open Label, Phase III Trial of Pazopanib versus Sunitinib in First-line Treatment of Patients with Metastatic Renal Cell Carcinoma (mRCC): Results of the COMPARZ Trial," Acta Oncologica, Vol. 23, No. 9S, 2012, Abstract No. LBA8_PR.

[26] C. Coppin, F. Porzsolt, A. Awa, J. Kumpf, A. Coldman and T. Wilt, "Immunotherapy for Advanced Renal Cell Cancer," Cochrane Database of Systematic Reviews, No. 1, 2005, Article ID: CD001425.

[27] J. J. Park, R. Omiya, Y. Matsumura, Y. Sakoda, A. Kuramasu, M. M. Augustine, S. Yao, F. Tsushima, H. Narazaki, S. Anand, Y. Liu, S. E. Strome, L. Chen and K. Tamada, "B7-H1/CD80 Interaction is Required for the Induction and Maintenance of Peripheral T-Cell Tolerance," Blood, Vol. 116, No. 8, 2010, pp. 1291-1298. doi:10.1182/blood-2010-01-265975

[28] R. H. Thompson, H. Dong, C. M. Lohse, B. C. Leibovich, M. L. Blute, J. C. Cheville and E. D. Kwon, "PD-1 is Expressed by Tumor-Infiltrating Immune Cells and is Associated with Poor Outcome For Patients With Renal Cell Carcinoma," Clinical Cancer Research, Vol. 13, No. 6, 2007, pp. 1757-1761. doi:10.1158/1078-0432.CCR-06-2599

[29] D. F. McDermott, C. G. Drake, M. Sznol, T. K. Choueiri, J. Powderly, D. C. Smith, J. Wigginton, D. McDonald, G. Kollia, A. K. Gupta and M. B. Atkins, "Clinical Activity and Safety of Anti-PD-1 (BMS-936558, MDX-1106) in Patients with Previously Treated Metastatic Renal Cell Carcinoma (mRCC)," Journal of Clinical Oncology, Vol. 30, 2012, Abstract No. 4505.

[30] S. L. Topalian, F. S. Hodi, J. R. Brahmer, S. N. Gettinger, D. C. Smith, D. F. McDermott, J. D. Powderly, R. D. Carvajal, J. A. Sosman, M. B. Atkins, P. D. Leming, D. R.
Spigel, S. J. Antonia, L. Horn, C. G. Drake, D. M. Pardoll, L. Chen, W. H. Sharfman, R. A. Anders, J. M. Taube, T. L. McMiller, H. Xu, A. J. Korman, M. Jure-Kunkel, S. Agrawal, D. McDonald, G. D. Kollia, A. Gupta, J. M. Wigginton and M. Sznol, "Safety, Activity, and Immune Correlates of Anti-PD-1 Antibody in Cancer," The New England Journal of Medicine, Vol. 366, No. 26, 2012, pp. 2443-2454. doi:10.1056/NEJMoa1200690

[31] B. I. Rini, J. Bellmunt, A. V. Alyzasova, J. Clancy, K. Wang, A. Niethammer and B. Escudier, "Randomized Phase IIIb Trial of Temsirolimus and Bevacizimab versus Interferon and Bevacizumab in Metastatic Renal Cell Carcinoma: Results from INTORACT," Annals of Oncology, Vol. 23, Suppl. 9, 2012, Abstract No. LBA21 PR.

[32] T. Hutson, B. Escudier, E. Esteban, G. A. Bjarnason, H. Y. Lim, K. Pittman, P. Senico, A. Niethammer, D. Lu, S. Hariharan and R. Motzer, "Temsirolimus vs. Sorafenib as Second-Line Therapy in Metastatic Renal Cell Carcinoma: Phase 3 Results from the INTORSECT Trial," Annals of Oncology, Vol. 23, Suppl. 9, 2012, Abstract No. LBA22 PR.

[33] R. J. Motzer, B. Escudier, P. Tomczak, S. Negrier, M. E. Gore, J. Tarazi, S. Hariharan, B. Rosbrook, S. Kim and B. I. Rini, "Axitinib vs Sorafenib for Advanced Renal Cell Carcinoma: Phase III Overall Survival Results and Analysis of Prognostic Factors," Annals of Oncology, Vol. 23, Suppl. 9, 2012, Abstract No. 793PD.

[34] D. Y. C. Heng, T. K. Choueiri, J. L. Lee, L. C. Harshman, G. A. Bjarnason, J. J. Knox, M. J. MacKenzie, U. N. Vaishampayan, T. Yuasa, M. H. Tan, S. Y. Rha, F. Donskov, N. Agarwal, S. K. Pal, C. K. Kollmannsberger, S. A. North, B. I. Rini and L. Wood, "An In-Depth Multicentered Population-Based Analysis of Outcomes of Patients with Metastatic Renal Cell Carcinoma (mRCC) That Do Not Meet Eligibility Criteria For Clinical Trials," Journal of Clinical Oncology, Vol. 30, 2012, Abstract No. 4536.

[35] C. Ferte, L. Albiges, J. C. Soria, Y. Loriot, K. Fizazi and B. J. Escudier, "The Use of Tumor Growth Rate (TGR) in Evaluating Sorafenib and Everolimus Treatment in mRCC Patients: An Integrated Analysis of the TARGET and RECORD Phase III Trials Data," Journal of Clinical Oncology, Vol. 30, 2012, Abstract No. 4540. 